Revista de Estudios Histórico-Jurídicos

[Sección historia del pensamiento jurídico]

XLI (Valparaíso, Chile, 2019)

[pp. 417 - 434 ]

\title{
MÁS ALLÁ DEL VOLUNTARISMO \\ DERECHO NATURAL EN EL PENSAMIENTO DE EMER DE VATTEL
}

[Beyond voluntarism. Natural law in Emer de Vattel's thought]

\author{
Fernando Pérez Godoy* \\ Pontificia Universidad Católica de Valparaíso, Chile
}

\begin{abstract}
RESUMEN
El presente artículo busca esclarecer la relación entre el pensamiento jurídico de Emer de Vattel y el voluntarismo en distintas obras del intelectual suizo hasta ahora poco atendidas en el ámbito iberoamericano. En este análisis teórico, se revalora la concepción de derecho natural de Vattel, tópico muchas veces olvidado en comparación con su famosa teoría sobre derecho de naciones. Se concluye que Vattel reprochó tempranamente un voluntarismo de corte protestante para continuar con esta posición epistemológica inicial al momento de abordar las relaciones internacionales y negocios de las naciones. Su voluntarismo representa más el producto de una construcción historiográfica moderna ligada al Estado-centrismo de la investigación internacional que una autentica expresión de su pensamiento legal.
\end{abstract}

\section{Palabras Clave}

Derecho de gentes - Emer de Vattel - voluntarismo - derecho natural moderno.

\section{Abstract}

The following paper explains the connection between the legal thought of Emer de Vattel and the voluntarism thorough different works which are rarely researched in the Ibero-American field. In this theoretical analyze, it revalues the Vattel's understanding of the natural law, a topic that is commonly under looked in compare with his law of nations' theory. It concludes that Vattel rejects early the protestant voluntarism in his natural law's conception, continuing with this epistemological view in his interpretation on the international relations of nations. His voluntarism represents more a historiographical modern construction tied to Statecentrism view in international scholarship than an authentic expression of his legal thought.

RECibIDO el 25 de abril de 2019 y ACEPTADO el 28 de junio de 2019

*Dr. phil. Johannes Gutenberg Universität Mainz. Alemania. Profesor Historia del Derecho, Pontificia Universidad Católica de Valparaíso, Chile. El presente artículo pertenece al Proyecto Fondecyt $\mathrm{N}^{\circ}$ 3180115. Email: fernando.perez@pucv.cl 


\section{INTRODUCCIÓN}

La relación de Emer de Vattel con el voluntarismo parece clara y resuelta ${ }^{1}$. Un rodeo por la historiografía jurídica deja claro la contribución del intelectual suizo al paradigma voluntarista positivista del derecho internacional público ${ }^{2}$. Georges Scelle lo nombra p ríncipe de los positivistas, toda vez que Vattel habría abierto la puerta al voluntarismo al sentar la dualidad de normas que regulan la conducta del Estado: el derecho natural y el derecho de gentes positivo ${ }^{3}$. Emmanuel Jouannet sostiene a su vez que Vattel es el primero en describir un concepto interestatal del derecho internacional dejando fuera la perspectiva interpersonal en la que el individuo queda relegado estrictamente a la esfera del Estado, negándole el estatus de sujeto de derecho internacional ${ }^{4}$. Por su cuenta, Rafael Domingo concuerda que Vattel inaugura el paradigma estatal y sustituye a la persona por el Estado en la praxis internacional'5 . Mary Ellen O'Connel resume mejor lo expuesto indicando que el derecho internacional en Vattel no es superior a los Estados, más bien, representa una herramienta útil entre Estados $^{6}$. Para D’Aspremont, Vattel es el primero en crear un sistema del derecho internacional en base a una concepción voluntarista, donde la moral cristiana o cualquier proyecto utópico está fuera del orden normativo internacional ${ }^{7}$.

El presente artículo quisiera ir en dirección opuesta a esta posición de la investigación internacionalista. Las afirmaciones expresadas antes están marcadas

\footnotetext{
${ }^{1}$ Dadas las numerosas definiciones de voluntarismo tomaremos de referencia la división básica entre un sistema que es regido por la voluntad (voluntas) y no por el intelecto. Voluntarism, en Encyclopaedia Britannica. Consultado en https://www.britannica.com/topic/voluntarismphilosophy. En el derecho internacional -sobre todo del siglo XIX-, seguimos la definición de Pellet: ius positum como ius voluntarium, reglas que están basadas en la sola voluntad de Estados, sin vinculo con la realidad social y que busca reducir científicamente al derecho a un cuerpo inmutable. Pellet, Alain, The Normative Dilemma: Will and Consent in International Law Making, en Australian Year Book of International Law, 12 (1992), p. 24.

${ }^{2}$ Pellet, Alain, cit. (n 1), p. 23.

${ }^{3}$ SCELle, Georges, Manuel de droit international public (Paris, 1949), p. 40.

${ }^{4}$ Jounannet, Emmanuelle, Emer De Vattel (1714-1767), en Fassbender, Bardo y Peters, Anne (eds.), The Oxford Handbook of the History of International Law (Oxford, 2012), pp. 1119- 1120. Hunter por su parte establece que Vattel es criticado tanto por su acento en el auto interés del Estado como por su idea iusnaturalista de justicia universal, siendo referencia para corrientes voluntaristas como para sus críticos. HunTER, Ian, Law, War, and Casuistry in Vattel's Jus Gentium, en Parergon, 28/2 (2011), p. 87.

${ }^{5}$ Domingo, Rafael, Gaius, Vattel, and the New Global Law Paradigm, en The European Journal of International Law, 22/3 (2011), pp. 635-637. Para Antony Carter no existiría la dicotomía entre práctica de Estados y doctrina en el derecho internacional, la práctica de Estados en las relaciones internacionales es una invención, una story, creada por los internacionalistas europeos a lo largo del siglo XIX. CARTY, Antony, Doctrine Versus State Practice, en Fassbender, Bardo y Peters, Anne (eds.), The Oxford Handbook of the History of International Law (Oxford, 2012), p. 976.

${ }^{6}$ O'Connell, Mary Ellen, Peace and War, en Fassbender, Bardo y Peters, Anne (eds.), The Oxford Handbook of the History of International Law (Oxford, 2012), p. 278.

${ }^{7}$ D'Aspremont, Jean, Formalism and the Sources of International Law A Theory of the Ascertainment of Legal Rules (New York, 2011), pp. 64-65.
} 
a nuestro entender por una visión historiográfica dogmática liberal que premia a Vattel por su contribución a la formación de la doctrina jurídica internacional contemporánea. Precisamente, su legado estaría en la descripción del orden internacional como un sistema universal de Estados igualmente autónomos y soberanos. En otras palabras, la relación Vattel y voluntarismo es parte de una visión historiográfica marcada por el relato de la secularización, universalización, profesionalización y cientificación del derecho internacional. Sin embargo, la nueva historia del derecho internacional, comola ha denominado Lauren Benton ${ }^{8}$, llama a deconstruir las European stories sobre ius gentium y sacar de tales narrativas esencialistas y lógicas euro- y Estado-céntricas a internacionalistas como Vattel.

En efecto, el derecho de gentes, establece Markus Kotzur ${ }^{9}$, gira no solo alrededor de un texto normativo y una dogmática, también, vive d e y e n sus narrativas. Esto quiere decir, como ha mostrado Jennifer Pitts, que el derecho de naciones descrito por Vattel como un sistema de Estados iguales y soberanos no tiene ninguna relación con la realidad de las relaciones exteriores y la política internacional de fines del siglo XVIII y sobre todo del siglo XIX ${ }^{10}$. Al contrario, el giro imperial de la historia del derecho internacional nos devela un o $\mathrm{r}$ d e $\mathrm{n}$ internacional racial y jerárquico gobernado por imperios y metrópolis y no por naciones del mismo rango compartiendo un sistema legal común ${ }^{11}$.

Junto con enfatizar el estudio histórico, ideológico y contextual ${ }^{12}$, el foco de la nueva historia del derecho internacional son las contradicciones e ideas entrecruzadas, anacrónicas, híbridas y regresivas que para la historiografía jurídica canónica -sobre todo decimonónica- se presentaron como errores, resultados no empíricos o meras contribuciones parciales a un proceso mejor logrado en el futuro $^{13}$. A mi entender, esta mentalidad se refleja también en el acento que pueda

\footnotetext{
${ }^{8}$ Benton, Lauren, Beyond Anachronism: Histories of International Law and Global Legal Politics, en Journal of History of International Law, 21 (2019), p. 11.

${ }^{9}$ Kotzur, Markus, Konstitutionelle Momente? Gedanke über den Wandel im Völkerrecht, en von ARnauld, Andreas (ed.), Völkerrechtsgeschichte(n) Historische Narrative und Konzepte im Wandel (Berlin, 2017), p. 99. CASSESE, Antonio, States: Rise and Decline of the Primary Subjects of the International Community, en FAssBender, Bardo y PeTERs, Anne (eds.), The Oxford Handbook of the History of International Law (Oxford, 2012), p. 52.

${ }^{10}$ PITTS, Jennifer, International relations and the critical history of International Law, en International Relations, 31/3 (2017), p. 287.

${ }^{11}$ PITTS, Jennifer, Boundaries of the International, Law and Empires (London, 2018), pp. 69-71.

${ }^{12}$ Olivares Jatib, Oscar y Pérez Godoy, Fernando, Historia y derecho internacional, en Revista de Estudios Histórico-Jurídicos, 40 (2018), pp. 141-165. KAPOssy, Béla, Rival Histories of Emer de Vattel's Law of Nations, en Grotiana, 31 (2010), pp. 11-13.

${ }^{13}$ No ponemos en duda la concepción de derecho natural como justicia, como predecesor del proceso de codificación jurídica ni su impacto en el desarrollo del constitucionalismo moderno, véase Carpintero Benítez, Francisco, Nuestros prejuicios acerca del llamado derecho natural, en Persona y Derecho, 27 (1992), pp. 101-112. Duve, Thomas, Entanglements in Legal History. Introductory Remarks, en ÉL Mismo, Entanglements in Legal History: Conceptual Approaches (Berlín, 2014), pp. 7-8.
} 
darse al derecho natural en obras como las del jurista suizo. En comparación con una historia del derecho de gentes entendida actualmente como clave para explicar el orden normativo internacional contemporáneo ${ }^{14}$, la historia del derecho natural y sus controversias parecen haber quedado rezagadas y desestimadas como oscuras querellas metafísicas de filósofos y teólogos en la historia. El derecho natural habría ganado importancia solo por experimentar procesos de diferenciación (Ausdifferenzierung) o desacralización (Entsakralisierung) ${ }^{15}$ de la tradición del ius naturae et gentium, posibilitando la nacionalización del derecho de gentes y confiando al estudio del ius naturae a una mera filosofía del derecho ${ }^{16}$. Tales conceptos terminaron condicionando también la historiografía jurídica donde solo el derecho de gentes pasará a ser parte de la historia del progreso del derecho occidental. No obstante, investigar la historia intelectual del derecho internacional no debe tener como objetivo per se la construcción de doctrina debido principalmente al actual problema del anacronismo y la contextualización ${ }^{17}$.

En este escrito, intentaremos ir más allá de la narrativa historiográfica que liga a Vattel con el voluntarismo, para preguntarnos, cuán efectiva es tal relación intelectual en escritos tempranos del internacionalista suizo. ¿Cuáles son los roles del voluntarismo y del derecho natural en su teoría jurídica? En lo siguiente, quisiéramos refrotar la tesis de que los juristas protestantes modernos -Grocio, Pufendorf, Wolff y el mismo Vattel- siguieron deduciendo el derecho de gentes a partir del derecho natural y por tal difícilmente asumieron una concepción puramente voluntarista del derecho internacional, como lo entenderán, por ejemplo, los internacionalistas positivistas del siglo XIX ${ }^{18}$. Si bien es sabido que los conceptos

${ }^{14}$ Para Steiger el desarrollo de la ciencia del derecho internacional, la diplomacia, el derecho de tratados, la historia del derecho de gentes en general, es la búsqueda de paz europea. STEIGER, Heinhard, Was heißt und zu welchem Ende studiert man Völkerrechtsgeschichte?, en ÉL MisMO (ed.), Universalität und Partikularität des Völkerrechts in geschichtlicher Perspektive. Aufsätze zur Völkerrechtsgeschichte 2008-2015 (Baden-Baden, 2015), pp. 51-62.

${ }^{15}$ Fiorillo, Vanda y Grunert, Frank, Einleitung, en Los Mismos (eds.), Das Naturrecht der Geselligkeit: Anthropologie, Recht und Politik im 18. Jahrhundert (Berlin, 2009), p. 5. SCHMIDT, Manfred, Wörterbuch zur Politik, (Stuttgart, 2010), p. 750. HAAKONSSEN, Knud, Natural law and moral philosophy: from Grotius to the Scottish Enlightenment (Cambridge, 1996). HOCHSTRASSER, Tim, Natural Law Theories in the Early Enlightenment (Cambridge, 2000). GIERKE, Otto, Natural Law and the Theory of Society 1500 to 1800 (trad. ingl., Boston, 1960).

${ }^{16}$ Carpintero Benítez, Francisco, La Cabeza de Jano (Cádiz, 1989), pp. 10-11.

${ }^{17}$ La metodología de Quentin Skinner de contextualizar textos e ideas, es decir, fijar estrictamente un marco espacio temporal determinado bajo el cual actores históricos son entendidos, choca con el anacronismo de juristas que buscando principios del derecho internacional para el presente trasgreden cronologías, periodificaciones y cánones del método histórico. Véase tal querella en BENTON, Lauren cit. (n. 8), pp. 3-11.

18 "Vattel's philosophy is therefore grounded in the natural law tradition, a fact which for some has been overlooked. His emphasis on sovereign consent, however, is widely regarded as marking a turning point in the evolution in international law. From the late eighteenth century onwards, international law is usually understood to be positive, not natural law. It is positive, not in the Austinian sense of being enacted by a superior but in being jointly willed by states, who bind themselves explicitly through treaties or implicitly through customary international law". RALPH, Jason, International Society-Consent and Custom as Sources of Law, en ÉL Mismo, Defending the Society of States (Oxford, 2007), p. 36. 
vattelianos de independencia e igualdad de los Estados, el principio de no intervención y la idea de una sociedad general humana son centrales para el desarrollo doctrinal del derecho internacional contemporáneo, intentaremos mostrar en este escrito una segunda lectura del pensamiento del jurista suizo que revalora su teoría iusnaturalista. Para tal fin, nos apoyaremos en lo que D’Aspremont denomina c o n c e p ci o n es híbrid a s del derecho internacional, fluctuantes entre la tradición del derecho natural y las tendencias voluntaristas, sin que aquello signifique caer un esquema de contradicciones. Estas tendencias híbridas son a nuestro entender más correctas para interpretar la historia del derecho natural y de gentes fuera del discutido marco historiográfico lineal, progresivo, euro y Estado-céntrico del proyecto de modernidad político jurídica ${ }^{19}$. Ha sido esta perspectiva canónica la que ha transmitido a nuestro entender la figura de Vattel ligada al voluntarismo positivista y sobrevalorado su rol como promotor de un sistema internacional compuesto por Estados igualmente soberanos y autónomos ${ }^{20}$.

\section{VATTEL COMO JURISTA GLOBAL}

La relación a estudiar propuesta entre voluntarismo y el pensamiento de Vattel se vuelve dificultosa si consideramos que su obra más difundida, Le droit des gens (1758), no constituye un tratado dogmático sobre la justicia, las leyes u obligaciones. Al contrario, es un manual práctico, dirigido, en palabras del mismo autor, a los soberanos y sus ministros ${ }^{21}$. El mismo Vattel, diplomático de profesión, menciona al inicio de su tratado que se ha separado de la aridez y fatiga que causaba la lectura de la obra de su maestro Christian Wolff ${ }^{2}$. Su objetivo, expresar lo más claro posible "verdades comunes" a todas las naciones ${ }^{23}$. Vattel fue discípulo de J.J. Burlamaqui y parte de la École romande du droit natural, pero sus referencias principales son Leibniz y la escolástica vía Christian Wolff ${ }^{4}$. Simplificar sus teorías sobre derecho natural y alcanzar un público mayor -di-

${ }^{19}$ Harris, Ron, Is it Time for Non-Euro-American Legal History?, en American Journal of Legal History, 56 (2016), p. 64.

${ }^{20}$ La división entre iusnaturalistas y voluntaristas, entre escolásticos y modernos-reformados, son solo categorías de las múltiples historias del derecho de gentes situadas en Europa. Estas historias adoptan el vocabulario europeo del progreso y la modernidad, las distinciones entre política y economía, entre religioso y secular, entre científico y místico, entre público y privado. Koskenniemi, Martii, Expanding Histories of International Law, en American Journal of Legal History, 104 (2016), p. 155.

21 "Le droit des gens est la loi des souverains. C'est pour eux principalement, \& pour leurs Ministres, qu'on doit l'écrire". VATtel, Emer de, Le droit des gens, ou Principes de la loi naturelle, appliqués à la conduite et aux affaires des Nations et des Souverains (2 vols, Londres, 1758), Préface, p. XXIII.

${ }^{22}$ Ibíd., p. XV.

23 "'Mais je n'ai point cru que, pour les démontrer, illatif toujours remonter jusques à leurs premiers fondements, \& je me fuis quelquefois contenter de les appuyer fur des Vérités communes, reconnues de tout lecteur de bonne-foi, fans pousser l'analyse plus loin". Ibíd., p. XXIII.

${ }^{24}$ Hochstrasser, Tim, Introduction, en Vattel, Emer de, The Law of Nations, Or, Principles of the Law of Nature, Applied to the Conduct and Affairs of Nations and Sovereigns, with Three Early Essays on the Origin and Nature of Natural Law and on Luxury (trad. ingl. 1797, 2008, Indianapolis, 1758), pp. x-xi. 
plomático- parece ser su desafío intelectual ${ }^{25}$. Intentando contextualizar las ideas vattelianas, Hunter explica que Vattel crea una concepción metafísica universal de derecho natural donde las naciones, como los hombres, buscan perfeccionarse unas a otras por medio de la comunicación y negocios internacionales-comercio, diplomacia-. Este principio usado por Vattel en su teoría del Estado, conlleva según Hunter la intención del suizo de realzar el Estado territorial protestante como una republica virtuosa y perfectible, cuyas expresiones históricas serían el cantón suizo y el Estado Principado de Sajonia ${ }^{26}$. Con el fin de asegurar la suerte de ambos Estados en el juego de poder imperial europeo del siglo XVIII, Hunter concluye que Vattel utiliza el principio de auto perfección de las naciones para ajustar el derecho a las circunstancias históricas de cada Estado y sus cambiantes políticas orientadas por el autointerés nacional. Al llegar a tal contextualización de su teoría, la particularidad del pensamiento vatteliano responde más a la experiencia histórica de la pequeña federación suiza que a un sistema de naciones soberanas.

Como hemos indicado, la imagen asociada a la obra de Vattel que remarca sus conceptos de nación, soberanía, igualdad jurídica y constitución sería atribuible a su posterior éxito a nivel global en academias, universidades, ministerios y tribunales en época de revoluciones y descolonización fuera del espacio europeo. Efectivamente, una mirada histórico global da cuenta de su amplia circulación y apropiación en espacios culturales, locales, diversos y distantes ${ }^{27}$. Ejemplo de la autoridad alcanzada por Vattel es la traducción de la obra Le droit de gens al chino. Lin Zexu, ministro inspector de la dinastía Qing, encargó el trabajo al medico misionero americano Peter Parker, apareciendo el texto en 1839 como 'Huada'er Geguo Luling'28. Una segunda traducción china fue realizada por Yuan Dehui y aparece en el compendio Haiguo Tuzhi de $1847^{29}$. Aunque ambas traducciones fueron imprecisas, su importancia estaba en que fueron hechas conforme a la moral y valores del confucianismo y en contexto de guerra del opio con el imperio británico. En 1907, el intelectual nipón S. Takahashi produjo su propio manual en japonés Heiji kokusaihôrom en base al texto del jurista suizo. En nuestro ámbito, aunque Vattel era un jurista prohibido por la Inquisición desde 1779, según el análisis de W. Hanisch, el manual vatteliano es utilizado desde la década de 1820 en el Instituto Nacional. Efectivamente, los resúmenes de Le Droit de Gens fueron publicados por el profesor Camilo Vial como texto de preparación de examen en $1826^{30}$. Luego, Andrés Bello utilizará este background para su curso de derecho de gentes en el Liceo de Chile. El programa de estudio de tal curso sería incluso

\footnotetext{
${ }^{25}$ NeFF, Stephan, Justice among Nations: A History of International Law (Cambridge, 2014), p. 196.

${ }^{26}$ Hunter, Ian, cit. (n 4), p. 90. Para Hunter finalmente el primado del derecho natural en las relaciones entre Estado se vuelve un idealismo impracticable.

${ }^{27}$ FIOCCHI, Elisabetta, L'eterno ritorno del Droit des gens di Emer de Vattel (secc. XVIII-XIX) (Frankfurt am Main, 2017), pp. 6-8.

${ }^{28}$ Kroll, Sebastian, Normgenese durch Re-Interpretation China und das europäische Völkerrecht im 19. und 20. Jahrhundert (Baden-Baden, 2012), p. 15.

${ }^{29}$ Kroll, Sebastian, cit. (n. 28), p. 87.

${ }^{30}$ Hanisch, Walter, Andrés Bello y el derecho natural, en Revista Universitaria, 6 (1981), p. 42.
} 
la base de sus Principios del Derecho de Gentes de $1832^{31}$. En la misma época, el intelectual y líder político liberal Francisco Javier Yanes resume a su vez los planteamientos de Vattel para las universidades de Nueva Granada ${ }^{32}$, mientras que Francisco de Miranda ya lo había citado en la Proclamación a los Pueblos del Continente Américo-Colombiano, del 2 de agosto de $1806^{33}$. Especialistas como Brian Richardson y Mark Graber discuten incluso el vatteleanismo de los padres fundadores, primeros congresistas y jueces norteamericanos ${ }^{34}$. Por su cuenta, José Carlos Chiaramonte ha dejado constancia de un uso parecido del texto en cuestión entre los criollos del Río de la Plata a fines de la colonia ${ }^{35}$. Un poco antes, a fines del siglo XVIII, José Portillo Valdés sostiene que cada español -de ambos hemisferios- con algún grado de instrucción podía citar la definición de nación propuesta por Vattel ${ }^{36}$. En todas estas partes del mundo, la obra del internacionalista suizo fue un repertorio simple y punto de acceso fluido a conceptos claves de derecho público moderno. No obstante, como hemos propuesto, es preciso también revalorizar su visión del derecho natural y con ello fijar el lugar efectivo del voluntarismo en su pensamiento.

\section{REVALORIZACIÓN DEL DERECHO NATURAL}

En consonancia con lo planteado, a nuestro entender, el tema de Vattel en su famoso tratado Le droit de gens del año 1758 no gira únicamente sobre el derecho de gentes ni sus distintas formas. Más bien, su reflexión sigue anclada en la tradición del derecho natural y en las antiguas querellas sobre su verdadero fundamento. Vattel lo expresa de otra forma: los principios del derecho natural que pueden aplicarse a los negocios de las naciones, precisamente, a las naciones

${ }^{31}$ Keller-Kemmerer, Nina, Die Mimikry des Völkerrechts “Andrés Bellos Principios de derecho internacional" (Baden-Baden, 2018), pp. 158-162.

${ }^{32}$ YANES, Francisco Javier, Idea general o principios elementales del derecho de gentes. Extracto de Vattel y otros autores (1824), en Él Mismo, Manual político del venezolano (Caracas, 1959).

${ }^{33}$ Miranda De, Francisco, Proclamación de don Francisco de Miranda, Comandante-General del Exército Colombiano, á los pueblos habitantes del continente Américo-Colombiano (Coro, 1806).

${ }^{34}$ Richardson, Brian, The Use of Vattel in the American Law of Nations, en The American Journal of International Law, 106/3 (2012), p. 547. Graber, Mark A. y Gillman, Howard, The complete American constitutionalism. Bd. 1, Introduction and colonial era (Oxford, 2015), pp. 101-105.

${ }^{35}$ Chiaramonte, José Carlos, Autonomía e independencia en el Río de la Plata, 1808-1810, en Historia Mexicana, 58/1 (2008), pp. 330-331. Portillo Valdés, José M., Crisis atlántica. Autonomia e independencia en la crisis de la monarquía hispana (Madrid, 2006), p. 176.

36 "Con el manual de Vattel como libro de cabecera, a Miranda no le costaba mucho argumentar sobre la nulidad e ineficacia jurídica de la bula de Alejandro VI, o sobre la injusticia de la conquista de los imperios aztecas e inca. En Vattel se podía aprender que el fundamento de la conquista y ocupación justa de tierras lejanas por naciones europeas descansaba en su incultura y falta de civilización". Portillo VAldÉs, José, cit. (n. 35), p. 175. Luego sostiene: "Literalmente era la definición que cualquier español con alguna formación intelectual aceptaba como de sentido común, y a la que los españoles de ambos hemisferios " pudieron leer trasladada a la Constitución que decía incluirlos y que proclamaba independencia y libertad como atributos esenciales de la nación y excluía del número de sus miembros, de los españoles, a aquellos que no eran libres al exigir esta condición como la primera para el reconocimiento de dicha cualidad de nacional". Ibíd., p. 173. 
pertenecientes al concierto de Estados europeos ${ }^{37}$. El suizo es claro en sentar que los Estados tienen y comparten offices d'humanité que provienen del mandato del derecho natural y más aún es esta normativa la que los obliga a formar una société humaine. Tal es el peso de este planteamiento que Vattel llega afirmar que el derecho de gentes, los tratados y usos de las naciones, un derecho internacional en base a la casuística en palabras de Hunter, son materia de la historia ${ }^{38}$ y no de un tratado sobre ius gentium ${ }^{39}$.

¿Qué entiende entonces el suizo por derecho natural? Una primera respuesta puede encontrarse en su disertación de 1742 titulada: ¿Puede traer el derecho natural perfección a la sociedad? En este escrito presentado en la Academia de Dijon, el intelectual oriundo de Neuchâtel indica que el derecho natural son las reglas de conducta basadas en la naturaleza de las cosas, particularmente, en la naturaleza humana ${ }^{40}$. Este derecho natural presenta las siguientes características: se conoce por medio de la razón, su creador es dios, es universal, eterno, inmutable, esencial al hombre y ninguna ley puede contradecirle ${ }^{41}$. Como ha establecido Hunter, es una definición netamente escolástica tomista que Vattel habría admitido por medio de su apego a la metafísica germana de Christian Wolff ${ }^{42}$. Según tal definición, el derecho natural consistiría en una serie de reglas a las cuales el hombre accede a través de la razón para la realización de su naturaleza perfectible. Como ser sociable y racional, tales reglas le permiten alcanzar una forma de vida justa y recta en sociedad. Un punto a destacar es que el suizo no especifica en su escrito cuáles son sus fuentes intelectuales. Un análisis del texto muestra que Vattel no cita pasajes de la biblia, ni el dogma reformado. Tampoco aparecen Occam, ni Santo Tomas, ni escolásticos ni algún otro filosofo medieval. Solamente nos encontramos con referencias a Cicerón, lo que acercaría su pensamiento a una teleología del hombre en base al concepto ciceroniano de felicidad ${ }^{43}$.

¿Cómo se deduce el derecho natural en Vattel? De la naturaleza humana se pueden deducir todos los deberes y obligaciones del derecho natural en la misma forma, aclara el internacionalista, que los matemáticos deducen las leyes del movimiento desde una simple proposición basada en la naturaleza de los cuerpos ${ }^{44}$.

${ }^{37}$ VATtel, Emer de, Le droit, cit. (n. 21), Preface, XIX.

${ }^{38}$ Hunter, Ian, Kant and Vattel in Context: Cosmopolitan Philosophy and Diplomatic Casuistry, en History of European Ideas, 39/4 (2013), pp. 477-502.

39 "Je dois me borner à donner les Principes généraux \& les règles, que la loi Naturelle fournit pour la direction des souverains à cet égard: le détail des différends Traités \& des diverses coutumes des Peuples appartient à l'Histoire, \& non pas à un Traité systématique du Droit de Gens". VATTEL, Emer de, Le droit, cit. (n. 21), p. XXII.

${ }^{40}$ Vattel, Emer de, Dissertation on This Question: Can Natural Law Bring Society to Perfection Without the Assistance of Political Laws?, en ÉL Mismo, The Law of Nations, Or, Principles of the Law of Nature, Applied to the Conduct and Affairs of Nations and Sovereigns, with Three Early Essays on the Origin and Nature of Natural Law and on Luxury (trad. ingl. 1797, 2008, Indianapolis, 1758), pp. 773-774.

${ }^{41}$ Ibíd., pp. 773-776.

${ }^{42}$ Hunter, Ian, cit. (n. 40), p. 89.

${ }^{43}$ VatTel, Emer de, Dissertation, cit. (n. 40), VII, p. 774.

44 "Indeed, from the nature of man can be deduced all the duties and commands of natural law, in the same way that mathematicians deduce all the laws of movement from this single proposition 
Luego, ¿pueden conocer todos los hombres el derecho natural y sus preceptos? Dada la corrupción del hombre, sus vicios en el intelecto, solo un grupo accede plenamente a su conocimiento (teoría reformada). Es más, Vattel plantea que si existiera una pequeña republica compuesta de los mil hombres más racionales y virtuosos de Europa, ¿quién podría dudar que esta sociedad no estaría mejor regulada solo por medio del derecho natural que cualquier otro Estado por el derecho político? Estos mil hombres, reflexiona el suizo, serían tan iluminados para conocer el derecho natural que formarían una sociedad perfecta, con un cuerpo jurídico justo y sabio, sin necesidad de autoridad y cuyo bien común nacería de la misma naturaleza ${ }^{45}$. Pero esta es una mera abstracción. Vattel concluye que, en el estado actual de la humanidad, el derecho natural por sí solo no lleva a la sociedad a la perfección y debe acudir a la asistencia del derecho civil. Como veremos más adelante, Vattel utilizará el concepto de Constitución como formula para fundar el derecho político en los preceptos del derecho natural ${ }^{46}$. Lo importante de esta primera definición, consiste en un principio metodológico clave de la teoría vatteliana: alcanzar el c o n o c i m i e n t o de la n a t u rale za de las cosas. Tal planteamiento epistemológico será clave para entender la relación de Vattel con respecto al voluntarismo.

\section{EL AUTO INTERÉS COMO FUNDAMENTO DEL DERECHO NATURAL}

Una mayor reflexión del suizo sobre el voluntarismo se encuentra en el escrito titulado: Ensayo sobre el fundamento de derecho natural y sobre el primer principio de la obligación de $1746^{47}$. Vattel sostiene en este escrito que si por el fundamento del derecho natural entendemos las fuentes por las cuales se derivan sus reglas y preceptos, se debe mirar la esencia y naturaleza del hombre y de las cosas en general $^{48}$. El suizo es claro en sentenciar que la ciencia del derecho natural es la que permite adquirir tal conocimiento confirmándose a posteriori o por experien$\mathrm{cia}^{49}$. Luego, Vattel denuncia aquellos que han errado en deducir las fuentes de la obligación y se pregunta: ¿Cómo llegaron a sus certezas los autores que algunas acciones son útiles a la raza humana y otras dañinas, sino es por la harmonía o discordancia de tales acciones con la naturaleza del hombre y de las cosas? ${ }^{50} \mathrm{De}$ tal razonamiento aparece su primer ataque a los voluntaristas denunciando que aquellos que asignan la institución del derecho natural a la voluntad arbitraria

based on the nature of bodies: "Every body remains in its state of rest or movement so long as no external force compels it to leave that condition”. Ibíd., VIII, p. 775.

${ }^{45}$ Ibíd., XIII, pp. 776-777.

${ }^{46}$ Ibíd, XVII-XX, pp. 779-780.

${ }^{47}$ Vattel, Emer de, Essay on the Foundation of Natural Law and on the First Principle of the Obligation Men Find Themselves Under to Observe Laws, en ÉL Mismo, The Law of Nations, Or, Principles of the Law of Nature, Applied to the Conduct and Affairs of Nations and Sovereigns, with Three Early Essays on the Origin and Nature of Natural Law and on Luxury (trad. ingl. 1797, 2008, Indianápolis, 1758).

${ }^{48}$ Ibíd., VI, p. 748.

${ }^{49}$ Ibíd.

${ }^{50}$ Ibíd., VI, p. 749. 
de Dios, deberían aceptar, que dios solo podría dar leyes favorables al bien de la sociedad y del individuo en particular. ¿Cómo tener aquella certeza? Vattel sostiene que aquello solo podríamos saberlo considerando la naturaleza de las cosas y particularmente la esencia y naturaleza del hombre ${ }^{51}$. Como señalamos, tal premisa epistemológica aparece una y otra vez en su reflexión jurídica.

Un punto importante a destacar en la teoría vatteliana, consiste en que el conocimiento de la naturaleza se realiza por medio de la razón, derivándose reglas generales y derechos que constituyen el cuerpo de la jurisprudencia natural ${ }^{52}$. Pero tales leyes no son suficientes que existan y sean conocidas. El hombre debe estar obligado a obedecerlas para que sean efectivas. Vattel reconoce que existe una gran discusión sobre el principio de esta obligación. Tal polémica nos es de gran utilidad porque revela su posición más crítica sobre el voluntarismo. Según el suizo, es posible encontrar tres líneas de interpretación que explican cómo se deduce el fundamento de la obligación: 1) la autoridad de un superior (dios como autor de la naturaleza). 2) la virtud. 3) el principio de sociabilidad de Grocio. Vattel responde que las obligaciones deben ser siempre morales y no forzadas por causa externa de un acto violento sobre el hombre. Así planteado, solo los $\mathrm{mot}$ ivos son razones suficientemente poderosas para llevar a los seres libres a actuar incluso contra sus inclinaciones físicas y pasiones ${ }^{53}$.

Siguiendo tal razonamiento, Vattel divide las obligaciones en activas y pasivas. Activa es la conexión del motivo con la acción. Obligación pasiva es la necesidad moral de hacer o no algo ${ }^{54}$. Su axioma es simple: no hay voluntad en el alma sin motivación ${ }^{55}$. Es la causa que produce el efecto. Para Vattel, ni Pufendorf ni Grocio habían definido qué se entiende por obligación pasiva y solo describían los efectos de la obligación -activa-. Obligación no es más que la conexión entre motivo y acción. Todo motivo actúa en concordancia con un deseo y el deseo básico es el a m o r p r o p i o (utile/utilité). Solo el amor propio, reflexiona el suizo, nos causa el deseo de buscar la perfección y felicidad tanto de nuestra condición interna -alma- como externa -cuerpo- ${ }^{-56}$. De este motivo general se desprenden todas las otras inclinaciones como el interés, el beneficio y el propio bienestar. En consecuencia, el principio de toda obligación es el i n t e r é $s^{57}$. No cabe duda, resume Vattel, que nuestro interés está vinculado a la observación de lo que el derecho natural nos enseña como naturalmente bueno o malo.

La sociabilidad que plantea Grotius como primer principio de la obligación es solo secundario. El hombre al entrar en sociedad y fundar el derecho civil aceptando su sumisión no renuncia a su propio interés, por el contrario, la

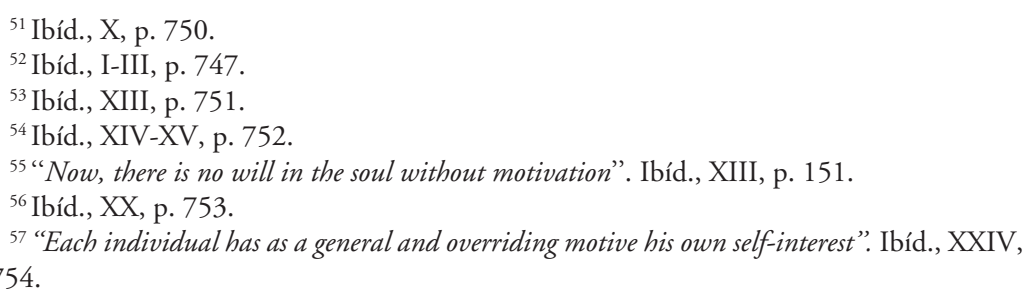


sociedad le trae mayores beneficios ${ }^{58}$. El traductor hugonote Jean Jack Barbeyrac había establecido que la voluntad de dios era la fuente de obligaciones y deberes. Vattel no rechaza de plano este principio, pero señala que los hombres estarían obligados por el derecho natural incluso fuera de la voluntad divina. El jurista suizo advierte que el interés propio no debe reducirse a la propiedad como algunos críticos mencionaban. Es un concepto más amplio que refiere a un noble auto interés condicionado por la observación, la práctica de virtud, la búsqueda de la perfección y la felicidad.

En todo este entramado argumentativo, el voluntarismo lo representa la posición de Barbeyrac aparecida en sus comentarios y citas a las obras de Grocio y Pufendorf. Vattel le reprocha principalmente el intento de asimilar obligación a sumisión y dependencia ${ }^{59}$. Un segundo error del voluntarismo de Barbeyrac consistía en confundir el fundamento de la obligación y su motivo. Para Vattel, Barbeyrac, a pesar de ingresar en un amplio análisis de la materia, no define cuál es el fundamento de la obligación ${ }^{60}$. Sin embargo, el suizo sostendrá que su posición puede reconciliarse con la del hugonote toda vez que obedecer la voluntad del legítimo superior siempre es bueno y aconsejable. Segundo, porque el superior puede recompensar o castigar, independiente del bien o mal que nuestra acción contiene naturalmente. Si el superior es sabio, su sola voluntad se impone sobre los hombres como una verdadera obligación, incluso si el hombre no supiera la integridad de la acción prescrita o si esta tiene o no relación con nuestra propia felicidad ${ }^{61}$. Solo en este caso, y por ello es tan importante la dimensión moral del buen gobernante, la voluntad toma el lugar de una buena razón (motivo). El problema, insiste Vattel, radica en que tal situación solo se puede dar con un superior todo poderoso y sabio (dios). Además, la posición de Barbeyrac de una voluntad divina tampoco explica la razón por la cual debemos obedecer la voluntad $^{62}$, aunque el suizo reconoce que aquello que la voluntad divina prescribe siempre es bueno y útil y deberíamos estar obligados a obedecerlo independiente si dios no lo ordenara ${ }^{63}$. A partir de Leibniz, concluye el internacionalista que la voluntad del legislador no es para los justos. Es decir, para aquellos que han alcanzado perfección en el alma -entendida como el conocimiento del fundamento de la ley natural- la voluntad sería finalmente incensaría ${ }^{64}$.

Merio Scattola ha descrito mejor el complejo debate intelectual sobre el derecho natural de los siglos XVII y XVIII, estableciendo que la escuela de Pufendorf, Barbeyrac y Thomasius habría extendido una definición voluntarista -hobbeseana- del derecho a la cual el joven Vattel por su acercamiento a Wolff

\footnotetext{
${ }^{58}$ Ibíd., XXV, p. 755.

${ }^{59}$ Ibíd., XVIII, p. 752.

60 "Expediency, he says, is the motive for obligation, it is not the foundation: we are required to obey a superior. But we may ask him: what, therefore, is the foundation of obligation, and for what reason am I to obey a superior?”. Ibíd., XXVII, p. 756.

${ }^{61}$ Ibíd., XXIX, p. 757.

${ }^{62}$ Ibíd., XXX, p. 761.

${ }^{63}$ Ibíd., XLI, p. 771.

${ }^{64}$ Ibíd., XLI, p. 771.
} 
y Leibniz rechazaba en muchos puntos. Compartiendo la conclusión de Scattola, la tradición del derecho natural no es la disputa ideológica por la antropología - pesimista u optimista- ni por el lugar de la teología en la disciplina. Al contrario, el gran debate gira en torno a la idea de que toda la experiencia y conducta humana puede finalmente ser descrita por medio de una racionalidad formal y reducida a un solo principio. Así, desde tal único factor, se puede desarrollar todo un sistema racional ${ }^{65}$. Como explicamos, Vattel pensó montar su teoría jurídica en la utilité. Esta postura epistemológica y metodológica utilizada para esclarecer el fundamento del derecho natural fue común a todas las tendencias, sean voluntaristas o intelectualistas, y corresponde para Scattola una verdadera continuidad en la premisa epistemológica que lleva a repensar el esquema tradicional que divide la historiografía jurídica de los siglos XVII y XVIII entre dos sistemas irreconciliables como la escuela voluntarista y la intelectualista.

\section{EL NUEVO SUJETO DEL IUS GENTIUM: NACIÓN COMO PERSONA MORAL}

La problemática a desarrollar por Vattel en su obra de 1758 es diferente. Su premisa es la siguiente: El derecho natural no se puede aplicar pura y simplemente a los Estados soberanos, necesita mudanzas ${ }^{66}$. El derecho natural descrito anteriormente como inmutable y universal sufriría cambios y mutaciones. Pero, ¿no sería tal planteamiento una contradicción a esta ley definida como eterna? Sin refutar los principios universalistas del ius naturae, el internacionalista suizo debía reformular el ámbito de investigación del ius gentium. Para ello, Vattel tiene a la vista los progresos de la ciencia europea y sus métodos ${ }^{67}$. Separa, segmenta, clasifica el conocimiento y el objeto de estudio. ${ }^{68}$ En su presente, reconoce siguiendo a Wolff, el derecho de gentes es ya una ciencia particular cuyas reglas son aplicadas a sujetos diversos y según la naturaleza de tales sujetos ${ }^{69}$. En este sentido, Vattel argumenta que la regla es solo aplicable de forma racional y justa cuando se sabe la n a t u r a l e z a d e l s u j e t o a aplicar. ¿Cuál es el sujeto en su pensamiento y la nature des nouveaux sujets? Si en su escrito de 1746 el sujeto de aplicación de la ley natural era la naturaleza humana, en su tratado mayor de 1758 será la nación. De ello se desprende que la ciencia del derecho de gentes tenga como objetivo la aplicación racional y recta de la ley natural a los asuntos y conductas

${ }^{65}$ Scattola, Merio, Scientia Iuris and Ius Naturae: The Jurisprudence of the Holy Roman Empire in the Seventeenth and Eighteenth Centuries, en Canale, Damiano et al. (eds.), A History of the Philosophy of Law in the Civil Law World, 1600-1900 (Heidelberg, 2009), p. 22.

66 "Une société Civile, un État, est un sujet bien différé d'un individu humain: D’ò̀ résultent, en vertu des Loi Naturelles mêmes, des obligations \& des droits bien différents, en beaucoup deçà la même règle générale, appliquée à deux sujets, ne pouvant opérer des décisions semblables, quand les sujets différents; ou une règle particulière, très juste pour un sujet, n'étaient point applicables à un fécond sujet de toute autre nature. II est donc bien des cas, dans lesquels la loi Naturelle ne décide point d'État à État, comme elles décideront de particulier à particulier"' VATTEL, Emer de, Le droit, cit. (n. 21), VI, p. 3.

${ }^{67}$ O`Connell, Mary Ellen, cit. (n. 6), p. 279.

${ }^{68}$ LandweHr, Achim, Kulturgeschichte (Stuttgart, 2009), pp. 74-87.

${ }^{69}$ VAtTel, Emer de, Le droit, cit. (n. 21), Preface, p. XII. 
de las naciones o los soberanos. Esto trae como consecuencia, aclara Vattel, que la ley natural deba mutar porque los Estados $-\mathrm{o}$ naciones- se conducen de un modo distinto que los particulares ${ }^{70}$.

Ahora bien, si la premisa metodológica de Vattel es conocer la naturaleza de las cosas: ¿Cuál es la naturaleza de la nación? La naturaleza de todas las naciones, lo que las iguala en su esencia, es la soberanía (par in parem non habet imperium). Importante para nuestro estudio resulta la afirmación de Vattel de que la sociedad política -nación- no parte de la voluntad de dios. En su teoría, nación y soberanía son expresiones propias de la voluntad de los hombres (un fait humain). La vida de la nación depende efectivamente de un a c to de a s o c i a c i ón en el cual cada miembro contrae la obligación común de asegurar el bien general, la seguridad y la defensa colectiva ${ }^{71}$. Así mismo, la conservation de la Nation, el deber más importante de una sociedad política, nace a partir de la voluntad humana expresada en un contrato social de los hombres ${ }^{72}$. No obstante, si la ley natural recomienda instaurar una sociedad civil a los hombres para su propia conservación, cediendo su libertad y autonomía natural, no ocurriría lo mismo con las naciones. La naturaleza, aclara Vattel, no les prescribe formar una civitas maxima como planteaba Wolff. Al contrario, les prescribe, como a los hombres, los derechos de igualdad y libertad, por tanto, viven en estado de naturaleza. ${ }^{73}$

Al no existir una civitas maxima o voluntad soberana de un legislador externo a las naciones, la única sociedad internacional que existe según su teoría es la sociedad de la naturaleza del género humano creada por el derecho natural ${ }^{74}$. En efecto, Vattel plantea un sistema en que la naturaleza crea un derecho de gentes voluntario y uno necesario -interno o de conciencia-. El derecho de gentes necesario es justo y bueno en sí, una ley sagrada que emana directo de la naturaleza y vincula a todos los hombres formando una s o c i e d a d ge n e ral de la h u m a n i d a d. Ningún pacto de las naciones puede cambiarle ni ninguna restarse de su normativa. Pero el derecho de gentes necesario, acá lo fundamental, es obligatorio siempre solo a nivel de conciencia del soberano ${ }^{75}$. De esta forma, el derecho natural -derecho gentes necesario- queda reducido a un c ó d i g o moral y solidaridad in ternacio nal sinsanción salvo el reproche

70 "Les États se conduisent autrement que des particuliers". Ibíd., p. XIX.

71 "Dans l'acte d'Association, en vertu duquel une multitude d'hommes forme ensemble un Etat, une Nation, chaque particulier est engagé envers tous à procurer le bien commun, \& tousse sont engagés envers chacun à lui faciliter les moyens de pourvoir à ses besoins, à le protéger \& à le défendre". Ibíd., Lib. I, Cap. II, XVI, p. 24.

72 "Cette obligation, naturelle aux individus que Dieu a -créés $r$ ne vient point aux Nations immédiatement de la Nature, mais du Pacte par lequel la société Civile est formée: aussi n'est-elle point absolue, mais hypothétique; c'est à-dire qu'elle suppose un fait humain, savoir le Pacte de Société ”. Ibíd., Lib. I, Cap. II, XVI.

${ }_{73}$ "Un Nain est aussi bien un homme, qu'un Géant : une petite République n'est pas moins un état souverain que le plus puissant Royaume”. Ibíd., XIX, p. 11.

74 "Je ne reconnais point d'autre Société naturelle entre les Nations, que celle-là même que la Nature a établie entre tous les hommes”. Ibíd., Preface, p. XVII.

${ }^{75}$ Hochstrasser advierte que tal conclusión del suizo le ha costado la constante critica de incoherente. HOCHSTRASSER, Tim, cit. (n. 24), p. xvi. 
moral. En cambio, el derecho de gentes voluntario abre la puerta al voluntarismo positivista. Vattel explica que solo en este caso el ius gentium es mutable y acepta cosas no necesariamente buenas ni justas, actos que el derecho natural condenaría pero que se to le r a n p o $\mathrm{n}$ e c e sidad. Como es conocido, tal derecho es voluntario porque depende del consentimiento de las naciones y se expresa por medio de pactos, tratados y convenios (derecho de gentes convencional) o por medio del consentimiento tácito (usos de los pueblos, costumbres de las naciones). Finalmente, tal derecho de gentes no es universal porque solo obliga a los que consienten o se hacen parte de sus usos.

Dada su importancia para nuestra investigación, queda por resolver: ¿Por qué la nación tiene voluntad? El concepto nación no aparece en el pensamiento de Pufendorf o Wolff. En Vattel se define como una forma colectiva en la que los hombres persiguen su perfección y felicidad. En el plano externo, la nación es una person a moral y por tanto posee intelecto y voluntad propia. Si la voluntad está ahora en lo que la nación quiere y no en un dios legislador o instancia normativa superior, Vattel debe precisar cuál es el objeto de la voluntad. Según su pensamiento, la voluntad de la nación es perfeccionarse a sí misma y para lograrlo necesita un aparato político que ejecute tal voluntad: el Estado soberano $^{76}$. Al llegar a este punto, el suizo agrega un concepto que será clave en el periodo de revoluciones políticas modernas. El ejercicio de la autoridad pública no se desarrolla sin una le y fu n d a m e n t a l que cada nación debe darse para asegurar y llenar los fines para los cuales se ha formado la asociación política. Por ello, Vattel no duda en establecer que el deber más importante de una nación para consigo misma es elegir la m e jor con s t i t u c ión pos i b l e yque más convenga a la índole de su pueblo y sus circunstancias ${ }^{77}$. Otros deberes de la nación son la práctica y fomento del comercio, la agricultura, la diplomacia y los negocios internacionales.

Hochstrasser ha indicado sobre este planteamiento que Vattel tendría la idea de una republica universal forjada por el deber de los Estados de asistirse unos a otros y aquello tendría su expresión más concreta en el desarrollo del comercio mundial y en la preponderancia de la economía política europea como factor de orden entre Estados. El derecho natural, al igual que a los individuos en estado de naturaleza, empujaría a las naciones a asistirse -comercialmente- unas a otras, pero al mismo tiempo, a limitar el comercio cuando este se volviera contrario a su utilite $^{78}$. Efectivamente, el internacionalista reconoce que la voluntad de la nación se ve condicionada por la obligación de unirse a otras naciones para alcanzar perfección y felicidad (fuente de la justicia). Pero esta no es una obligación universal. Cada nación decide qué es justo, por ello, el derecho de gentes voluntario puede cometer injusticia y no coincidir con el derecho natural. Solo al llegar a este punto podemos sustentar que el pensamiento del intelectual suizo adhiere al voluntarismo. El derecho natural, sus preceptos a nivel de la comunidad

\footnotetext{
${ }^{76}$ Vattel, Emer de, Le droit, cit. (n. 21), XXI, p. 27.

${ }^{77}$ Ibíd., XXVIII, p. 46.

${ }^{78}$ Hochstrasser, Tim, cit. (n. 24), p. xiv-xv.
} 
internacional, dependerán de la voluntad del soberano ${ }^{79}$. Bajo la misma premisa, Vattel concluye que no existe justicia entre las naciones, solo reglas claras para una guerra regulada o disputas neutralizadas. Finalmente, Neff ha precisado que Vattel sigue a Wolff al establecer que el derecho de gentes más que ser de naturaleza voluntario, sería o p c i o n a l y dependiente del c o n s e n t i m i e n t o de cada nación ${ }^{80}$. Así planteado, el derecho de gentes voluntario en Vattel es un conjunto de reglas de conducta que el derecho natural obliga a toda nación a consentir, de modo que cuando alguna nación rehúsa hacerlo, está violando la sociedad natural de la humanidad ${ }^{81}$.

\section{CONCLUSIÓN}

Concluimos que el rechazo de Emer de Vattel al voluntarismo es evidente en sus escritos de juventud de 1742 y 1746 . En tales textos, el suizo es claro en sentar que el contenido de la ley natural no puede ser fijado ni por la voluntad del legislador como tampoco lo puede hacer la voluntad de dios. Su principal opositor será Jean Jack Barbeyrac. Vattel sostiene que el motivo antecede a la voluntad y el auto interés (motivo principal) representa la fuente de toda obligación del hombre. Como la nación es una obra humana, es lógico que el jurista entienda que el auto interés de la nación -auto perfección- sea el deber principal del ius gentium. Sin embargo, hemos remarcado que el intelectual suizo nunca abandona la idea iusnaturalista universalista al sentar la noción de una sociedad general de la humanidad basada en buenos oficios nacidos del derecho natural. Esto es muy distinto a la común opinión historiográfica en que el orden internacional en Vattel representa un sistema de Estados soberanos e iguales buscando su auto interés sin ningún tipo de regulación moral. En ningún caso, como hemos visto, Vattel sostendrá que la ley natural es la expresión de la mera voluntad del legislador ni encontramos en su obra la imagen historiográfica de un Estado libre de todo límite, sirviente a su propio interés y viviendo en la más abstracta soledad, lo que Nietzsche llama el más frío de todos los fríos monstruos. En nuestro análisis ha sido necesario precisar que los preceptos del derecho natural no desaparecen de su teoría, pero quedan a criterio opcional del consentimiento soberano. Solo entonces es posible hablar de voluntarismo en Vattel.

Por otro lado, como hemos advertido en base a los avances de la nueva historia del derecho internacional, la experiencia histórica del siglo XVIII, sobre todo el contexto intelectual, político y cultural de Vattel, se distanciaría de la narrativa de un orden internacional moderno compuesto por Estados soberanos y donde no existen poderes supranacionales -imperios, papado, sultán, civitas maxima-, como tampoco fundamentos metafísicos - republica cristiana-. En efecto, Vattel plantea la idea de independencia de las naciones de una instancia político-jurídica externa

\footnotetext{
${ }^{79}$ Vedross establece que el principio de independencia de los Estados no significa independencia de las normas de la moral y del derecho de gentes, sino solo independencia de un orden jurídico externo. Vedross, Alfred, Völkerrecht (Viena, 1955), p. 7.

${ }^{80}$ NefF, Stephan, cit. (n. 25), p. 196.

${ }^{81}$ Ibíd., p. 197.
} 
de soberanía universal, pero no de una normativa común al género humano. Este planteamiento sería atribuible a su propio contexto histórico, donde la federación suiza y el Principado de Sajonia, dos de los pequeños Estados a los que Vattel sirvió como funcionario, debían enfrentar el entramado de poder europeo de fines del siglo XVIII sin la regulación de un orden normativo internacional consolidado.

Un estudio más detallado de la doctrina y del pensamiento jurídico de Vattel, invita a repensar a su vez las tajantes separaciones de la historiografía clásica entre derecho natural y derecho de gentes o las contraposiciones entre intelectualismo y voluntarismo. Como quisiéramos concluir, una idea pura y esencialista del concepto de derecho de gentes no sería sustentable desde el punto de vista histórico jurídico global. Siguiendo tal perspectiva, hemos querido ejemplificar los desplazamientos de significado que experimentó su tratado Le droit de gens al circular en espacios extra europeos. En la práctica, China, Japón o Latinoamérica en el siglo XIX no formaban parte de un sistema de Estados iguales y soberanos, más bien, en tales espacios luchaban formas no tradicionales de Estado-Nación, colonias, imperios, quasi soberanías y Estados de facto. Así planteado, los conceptos del derecho de gentes no deben medirse necesariamente por su estabilidad, lógica e inmutabilidad sino por los desplazamientos de significados que experimentan en cada contexto, espacio y tiempo.

BiBLIOGRAFÍA

BENTON, Lauren, Beyond Anachronism: Histories of International Law and Global Legal Politics, en Journal of History of International Law, 21 (2019), pp. 1-34.

Carpintero Benítez, Francisco, Nuestros prejuicios acerca del llamado derecho natural, en Persona y derecho, 27 (1992), pp. 21-200.

Carty, Antony, Doctrine Versus State Practice, en Fassbender, Bardo y Peters, Anne (eds.), The Oxford Handbook of the History of International Law (Oxford, Oxford University Press, 2012), pp. 973-997.

CASSESE, Antonio, States: Rise and Decline of the Primary Subjects of the International Community, en Fassbender, Bardo y Peters, Anne (eds.), The Oxford Handbook of the History of International Law (Oxford, 2012), pp. 51-71.

CoING, Helmut, Das Recht als Element der europäischen Kultur, en Historische Zeitschrift, 238 (1984), pp. 1-16.

Chiaramonte, José Carlos, Autonomía e independencia en el Río de la Plata, 18081810, en Historia Mexicana, 58/1 (2008), pp. 325-368.

D'Aspremont, Jean, Formalism and the Sources of International Law A Theory of the Ascertainment of Legal Rules (New York, Oxford University Press, 2011).

Domingo, Rafael, Gaius, Vattel, and the New Global Law Paradigm, en The European Journal of International Law, 22/3 (2011), pp. 627-647.

DuchHARDT, Heinz, >>Friedensvermittlung $<<$ im Völkerrecht des 17. und 18. Jahrhunderts: Von Grotius zu Vattel, en EsPENHORST, Martin, Frieden im Europa der Vormoderne Ausgewählte Aufsätze 1979-2011 (Paderborn, Ferdinand Schöningh, 2012), pp. 26-45.

Duve, Thomas, Entanglements in Legal History. Introductory Remarks, en ÉL Mismo, Entanglements in Legal History: Conceptual Approaches (Berlín, Max Planck Institut für europäische Rechtsgeschichte, 2014), pp. 3-25. 
FIOCCHI, Elisabetta, L'eterno ritorno del droit des gens di Emer de Vattel (secc. XVIII-XIX) (Frankfurt am Main, Max Planck Institut für europäische Rechtsgeschichte, 2017). Fiorillo, Vanda y Grunert, Frank, Einleitung, en Los Mismos (eds.), Das Naturrecht der Geselligkeit: Anthropologie, Recht und Politik im 18. Jahrhundert (Berlin, Duncker \& Humblot, 2009), p. 55-68.

Gierke, Otto, Natural Law and the Theory of Society 1500 to 1800 (trad. ingl., Boston, Cambridge University Press, 1960).

Graber, Mark A. y Gillman, Howard, The complete American constitutionalism. Bd. 1, Introduction and colonial era (Oxford, Oxford University Press, 2015).

HaAKOnssen, Knud, Natural law and moral philosophy: from Grotius to the Scottish Enlightenment (Cambridge, Cambridge University Press, 1996).

Hanisch, Walter, Andrés Bello y el derecho natural, en Revista Universitaria, 6 (1981), pp. 38-56.

Harris, Ron, Is it Time for Non-Euro- American Legal History?, en American Journal of Legal History, 56 (2016), pp. 60-65.

Hochstrasser, Tim, Natural Law Theories in the Early Enlightenment (Cambridge, Cambridge University Press, 2000).

- Introduction, en Vattel, Emer de, The Law of Nations, Or, Principles of the Law of Nature, Applied to the Conduct and Affairs of Nations and Sovereigns, with Three Early Essays on the Origin and Nature of Natural Law and on Luxury (trad. ingl. 1797, 2008, Indianapolis, Liberty Fund, 1758), pp. ix-xx.

Hunter, Ian, Law, War, and Casuistry in Vattel's Jus Gentium, en Parergon, 28/2 (2011), pp. 87-104.

- Kant and Vattel in Context: Cosmopolitan Philosophy and Diplomatic Casuistry, en History of European Ideas, 39/4 (2013), pp. 477-502.

JouAnnet, Emmanuelle, Emer De Vattel (1714-1767), en Fassbender, Bardo y Peters, Anne (eds.), The Oxford Handbook of the History of International Law (Oxford, Oxford University Press, 2012), pp. 1119- 1122.

Kapossy, Béla, Rival Histories of Emer de Vattel's Law of Nations, en Grotiana, 31 (2010), pp. 5-21.

Keller-Kemmerer, Nina, Die Mimikry des Völkerrechts "Andrés Bellos Principios de derecho internacional" (Baden-Baden, Nomos Verlag, 2018).

Koskenniemi, Martii, Expanding Histories of International Law, en American Journal of Legal History ,104 (2016), pp. 104-112.

- Histories of International law: Dealing with Eurocentrism, en Legal History, 19 (2011), pp. 152-176.

Kotzur, Markus, Konstitutionelle Momente? Gedanke über den Wandel im Völkerrecht, en VON ARNauld, Andreas (ed.), Völkerrechtsgeschichte(n) Historische Narrative und Konzepte im Wandel (Berlin, Duncker \& Humblot, 2017), pp. 100-118.

Kroll, Sebastian, Normgenese durch Re-Interpretation China und das europäische Völkerrecht im 19. und 20. Jahrhundert (Baden-Baden, Nomos Verlag, 2012).

Voluntarism, Encyclopaedia Britannica. Consultado en https://www.britannica.com/ topic/voluntarism-philosophy.

LandweHr, Achim, Kulturgeschichte (Stuttgart, UTB, 2009).

Miranda De, Francisco, Proclamación de don Francisco de Miranda, ComandanteGeneral del Exército Colombiano, á los pueblos habitantes del continente AméricoColombiano (Coro, 1806). 
NefF, Stephan, Justice among Nations: A History of International Law (Cambridge, Cambridge University Press, 2014).

O'Connell, Mary Ellen, Peace and War, en Fassbender, Bardo y Peters, Anne (eds.), The Oxford Handbook of the History of International Law (Oxford, Oxford University Press, 2012), pp. 273-295.

Olivares Jatib, Oscar y Pérez Godoy, Fernando, Historia y derecho internacional, en Revista de Estudios Histórico-Jurídicos, 40 (2018), pp. 141-165.

Pellet, Alain, The Normative Dilemma: Will and Consent in International Law Making, en Australian Year Book of International Law, 12 (1992), pp. 22-53.

PIтTS, Jennifer, Boundaries of the International, Law and Empires (London, Harvard University Press, 2018).

Portillo Valdés, José M., Crisis atlántica. Autonomía e independencia en la crisis de la monarquia hispana (Madrid, Marcial Pons, 2006).

RaLPH, Jason, International Society-Consent and Custom as Sources of Law, en ÉL Mismo, Defending the Society of States (Oxford, 2007), pp. 29-55.

Richardson, Brian, The Use of Vattel in the American Law of Nations, en The American Journal of International Law, 106/3 (2012), pp. 547-571.

Scattola, Merio, Scientia Iuris and Ius Naturae: The Jurisprudence of the Holy Roman Empire in the Seventeenth and Eighteenth Centuries, en Canale, Damiano et al. (eds.), A History of the Philosophy of Law in the Civil Law World, 1600-1900 (Heidelberg, Springer, 2009), pp. 1-39.

SCELLE, Georges, Manuel de droit international public (Paris, Domat- Montchreisten, 1948).

SchmidT, Manfred, Wörterbuch zur Politik, (Stuttgart, Kröner, 2010).

SKINNER, Quentin, Los fundamentos del pensamiento politico moderno, II: La Reforma (trad. cast. 1986, México D.F., 1978).

STEIGER, Heinhard, Was heißt und zu welchem Ende studiert man Völkerrechtsgeschichte?, en ÉL Mismo (ed.), Universalität und Partikularität des Völkerrechts in geschichtlicher Perspektive. Aufsätze zur Völkerrechtsgeschichte 2008-2015 (Baden-Baden, Nomos Verlga, 2015), pp. 49-62.

VATTEL, Emer de, Le droit des gens, ou Principes de la loi naturelle, appliqués à la conduite et aux affaires des Nations et des Souverains (2 vols, Londres, 1758).

- Dissertation on This Question: Can Natural Law Bring Society to Perfection Without the Assistance of Political Laws?, en ÉL Mismo, The Law of Nations, Or, Principles of the Law of Nature, Applied to the Conduct and Affairs of Nations and Sovereigns, with Three Early Essays on the Origin and Nature of Natural Law and on Luxury (trad. ingl. 1797, 2008, Indianapolis, Liberty Fund, 1758), pp. 773-782.

- Essay on the Foundation of Natural Law and on the First Principle of the Obligation Men Find Themselves Under to Observe Laws, en ÉL Mismo, The Law of Nations, Or, Principles of the Law of Nature, Applied to the Conduct and Affairs of Nations and Sovereigns, with Three Early Essays on the Origin and Nature of Natural Law and on Luxury (trad. ingl. 1797, 2008, Indianápolis, Liberty Fund, 1758), pp. 745-772.

Vedross, Alfred, Völkerrecht (Viena, Springer, 1955).

YANES, Francisco Javier, Idea general o principios elementales del derecho de gentes. Extracto de Vattel y otros autores (1824), en ÉL Mismo, Manual político del venezolano (Caracas, Biblioteca de la Academia Nacional de la Historia, 1959). 